\title{
Spin Correlation and W Helicity in Top Events with ATLAS
}

\section{Markus Jüngst*}

On behalf of the ATLAS Collaboration

CERN

E-mail: ralph.markus.jungstecern.ch

In $7 \mathrm{TeV}$ proton-proton collisions at the LHC, pairs of top and anti-top quarks are thought to be mostly produced through gluon fusion, in contrast to production at the Tevatron, where quarkantiquark annihilation dominates. The ATLAS experiment has now recorded a large number of top quark pair events, allowing this process to be explored in detail. Measurements of the spin correlation between top and anti-top quarks as well as measurements of the $\mathrm{W}$ helicity fractions from top quark decays are presented. Both values are precisely predicted by the Standard Model and a deviation could reveal the presence of new physics.

36th International Conference on High Energy Physics,

July 4-11, 2012

Melbourne, Australia

\footnotetext{
* Speaker.
} 


\section{Introduction}

The top quark was discovered in 1995 [1,2] at the Fermilab Tevatron collider and has a measured mass of $173.2 \pm 0.9 \mathrm{GeV}$ [3]. Due to their high mass, top quarks have another remarkable property, their short lifetime of about $5 \times 10^{-25}$ seconds. This is at least an order of magnitude shorter than the time-scale for strong interactions, implying that the top quark decays before hadronisation. Thus the top quark does not form bound states before its decay, allowing the study of a bare quark [4]. While top quarks produced in pairs at hadron colliders are unpolarised, their spins are correlated. Top quarks are predicted to decay via the electroweak interaction into a $W$ boson and a bottom quark with a nearly $100 \%$ branching fraction. Events with $t \bar{t}$ pairs can thus be classified according to the decays of the two produced $W$ bosons. Each boson can decay either into a quark-antiquark pair or into a charged lepton and a neutrino. This note summarizes the result of two top quark property measurements. In the first part, the analysis of spin correlation in the $t \bar{t}$ system, which is transferred to the decay products as measured directly via their angular distributions [5], is shown [6]. In the second part a description is given of the polarization measurement [7] of the $W$ boson originating from top decays in $t \bar{t}$ events, probing the $(\mathrm{V}-\mathrm{A})$ structure of the $W t b$ vertex.

\section{Spin Correlation}

A measurement of the spin correlation tests the predictions of QCD such as whether the decay of the top quark occurs before its spin is flipped by the strong interaction [8]. The apparent spin correlation may differ from that expected in the Standard Model (SM) due to sources of physics beyond the SM in the production of top quark pairs or in their decay.

The different beams and centre-of-mass energy make measurements of the spin correlation at the Tevatron [9] and LHC colliders complementary. In top quark decays in the SM, the VA couplings fix the angular distribution of the decay products according to the polarisation of the parent top quark. Charged leptons are the most effective spin analysers, since they carry the full information concerning the spin of the parent top quark at leading order. Therefore, here the dilepton final states is analysed.

The spin correlation coefficient, $A$, is defined as the difference in the fraction in the number of events where top quark spins are aligned and where they have opposite alignment,

$$
A=\frac{N_{\text {same }}-N_{\text {opposite }}}{N_{\text {same }}+N_{\text {opposite }}}=\frac{N(\uparrow \uparrow)+N(\downarrow \downarrow)-N(\uparrow \downarrow)-N(\downarrow \uparrow)}{N(\uparrow \uparrow)+N(\downarrow \downarrow)+N(\uparrow \downarrow)+N(\downarrow \uparrow)},
$$

where the arrows denote the spins of the top and antitop quarks with respect to a quantisation axis. It has been shown [10] that the $\Delta \phi$ distribution between the two leptons in the laboratory frame is sensitive to the spin correlation.

$A$ is derived in both the helicity and the LHC maximal bases [11] by fitting the observed $\Delta \phi$ distribution to a linear superposition of that expected from the SM spin correlation, and that expected with no spin correlation. A binned likelihood fit is used to extract the correction factor to the SM template $\left(f_{\mathrm{SM}}\right)$, which measures the deviation from the SM expectation and is balanced by a complementary contribution from the zero correlation template. This correction factor was 

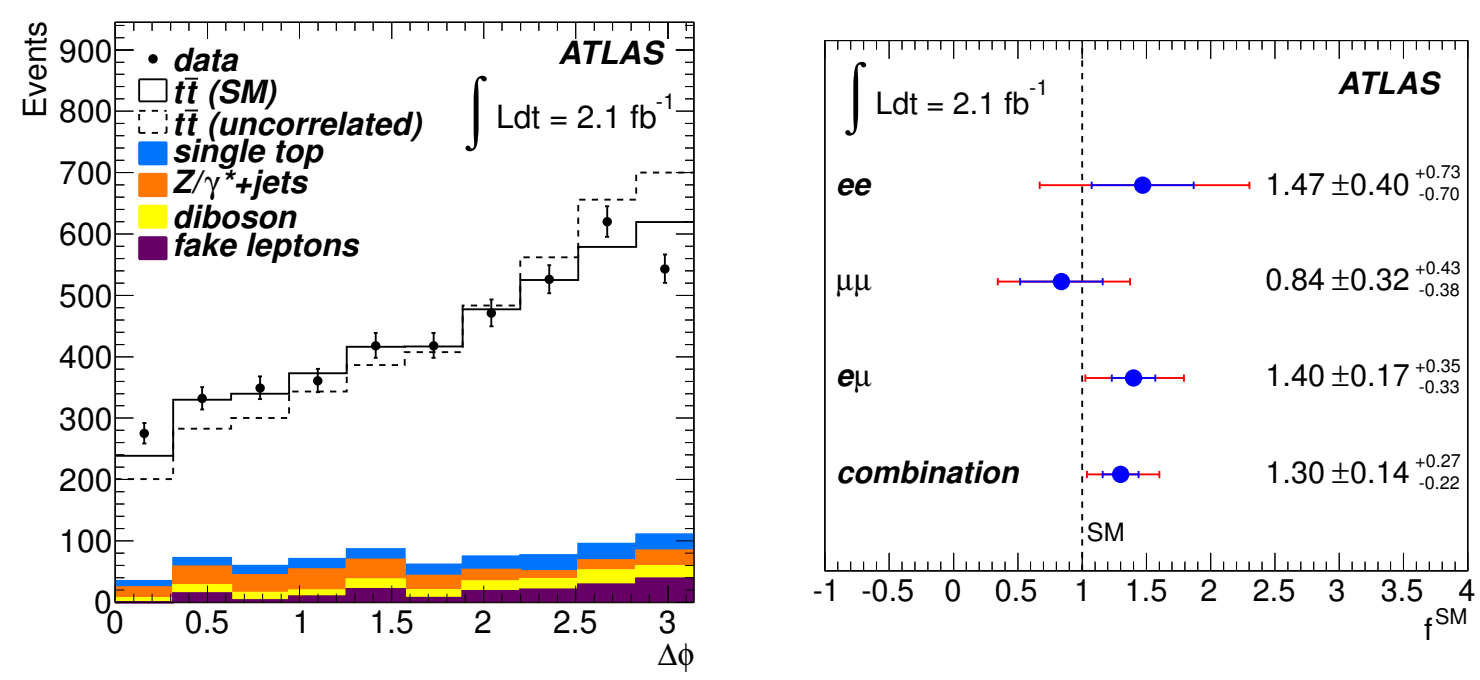

Figure 1: (Left) Reconstructed charged lepton $\Delta \phi$ distribution for the sum of the three dilepton channels. The white histograms show the MC sum using the default $t \bar{t}$ sample (solid line) and the sample with no spin correlation (dashed line). (Right) Correction parameter $f_{S M}$ for the three dilepton channels and from the combined fit. The error bars indicate the statistical and systematic uncertainties [6].

determined in the three dilepton subchannels $(e e, \mu \mu$ and $e \mu)$ separately as well as in a combined fit of the three channels. A detailed description of the event selection can be found in Ref. [6].

Several sources of uncertainties are considered. The dominant uncertainties arise from the jet energy scale (including uncertainties in the flavour composition, the presence of nearby jets and additional uncertainties due to pileup) and resolution and from shape uncertainties of the background templates (mainly from the multijet background).

Figure 1 (left) shows the reconstructed $\Delta \phi$ distribution for the sum of the three dilepton channels. The simulation is able to reproduce the shape of the data very well. Figure 1 (right) summarizes the correction factor from the best fit for the three dilepton channels separately and for the combined fit. The measured spin correlation from the latter is $f_{\mathrm{SM}}=1.30 \pm 0.14(\text { stat })_{-0.22}^{+0.27}($ syst $)$. All fits are in agreement within their uncertainties with the SM prediction $\left(f_{\mathrm{SM}}=1\right)$.

The correlation coefficient in any basis can then be directly calculated by the product of the predicted coefficient and the correction factor. This yields a measured correlation coefficient of $A_{\text {helicity }}=0.40 \pm 0.04_{-0.07}^{+0.08}$ and $A_{\text {maximal }}=0.57 \pm 0.06_{-0.10}^{+0.12}$ for the helicity and the maximal bases, respectively. In the helicity basis the $\mathrm{SM}$ prediction is $A_{\mathrm{SM}}=0.32$, evaluated at parton helicity level using MC@NLO with the CTEQ6.6 parton distribution function (PDF) [12]. The $\mathrm{SM}$ expectation is evaluated in the same way for the maximal basis and is found to be $A_{\mathrm{SM}}=0.44$. This measurement of $t \bar{t}$ spin correlations using $2.1 \mathrm{fb}^{-1}$ of ATLAS data is inconsistent with a hypothesis of zero spin correlation with a significance of 5.1 standard deviations.

\section{W Boson Polarization}

The $W t b$ vertex is an electroweak interaction and has $(\mathrm{V}-\mathrm{A})$ structure. Since $W$ bosons are produced as real particles in top quark decays, their polarization can be longitudinal, left-handed 

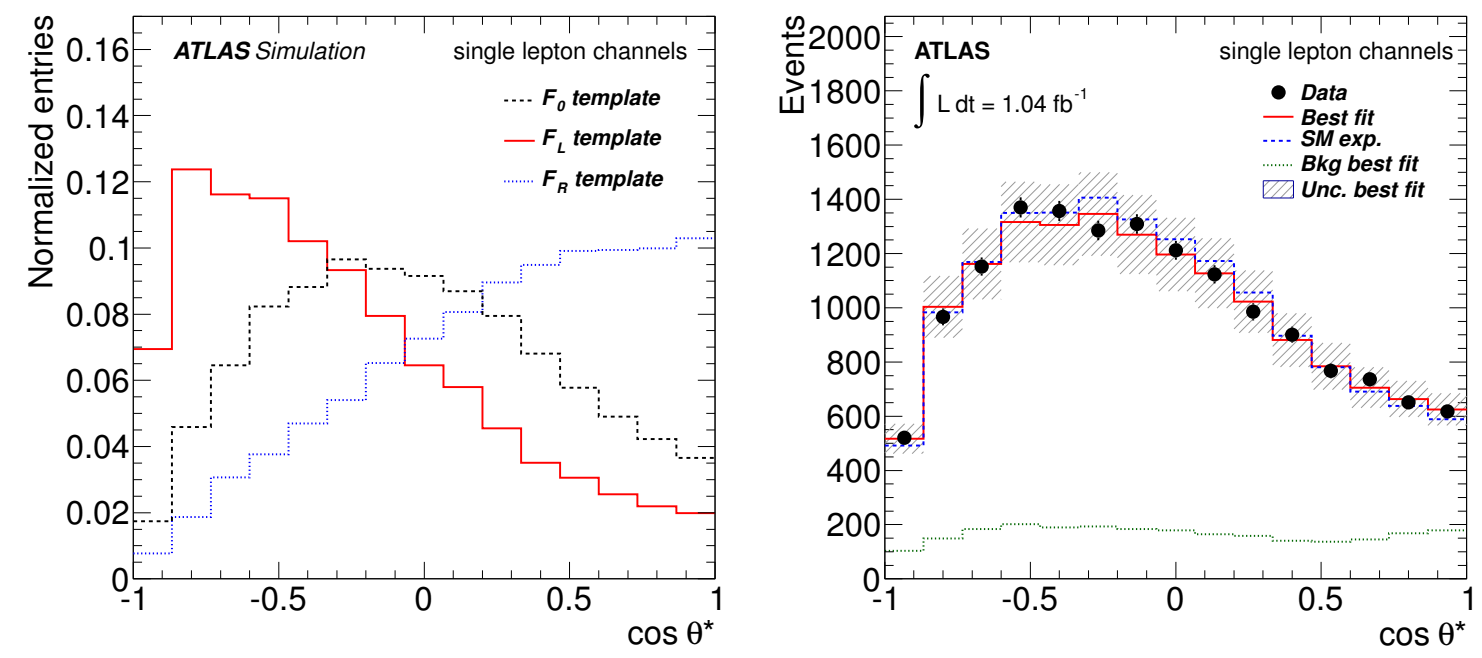

Figure 2: (Left) Distributions of $\cos \Theta^{*}$ for each of the three simulated signal templates and (right) of the reconstructed $\cos \Theta^{*}$ for data (markers), fitted background (dotted line), the Standard Model prediction (dashed line) and the distribution using the best fit values for the $F_{0}, F_{L}$ and $F_{R}$ contributions plus background contributions (solid line) [7].

or right-handed. The fractional contributions can be extracted from measurements of the angular distribution of the decay products of the top quark [13]:

$$
\frac{1}{\sigma} \frac{\mathrm{d} \sigma}{\mathrm{d} \cos \Theta^{*}}=\frac{3}{4}\left(1-\cos ^{2} \Theta^{*}\right) F_{0}+\frac{3}{8}\left(1-\cos \Theta^{*}\right)^{2} F_{L}+\frac{3}{8}\left(1+\cos \Theta^{*}\right)^{2} F_{R},
$$

where the angle $\Theta^{*}$ is defined as the angle between the momentum direction of the lepton and the reversed momentum direction of the b-quark from the top decay, both boosted into the $W$ boson rest frame. These helicity fractions can be calculated at next-to-next-to-leading-order (NNLO) in QCD yielding $F_{0}=0.687 \pm 0.005, F_{L}=0.311 \pm 0.005, F_{R}=0.0017 \pm 0.0001$ [14].

The measurements of the $W$ boson polarization presented here are based on data collected with the ATLAS detector [15] in 2011 corresponding to an integrated luminosity of $1.04 \mathrm{fb}^{-1}$. In this case both the single-lepton and dilepton decay topologies are considered and combined for the final result. The helicity fractions are obtained from a combination of template fits to the reconstructed $\cos \Theta^{*}$ distributions, and angular asymmetries calculated from the unfolded $\cos \Theta^{*}$ distribution.

For the first method in the single lepton channel templates for different signal and background processes are fitted to the observed $\cos \Theta^{*}$ distributions based on events reconstructed with a kinematic fit in the single-lepton channels [18]. Figure 2 (left) shows the normalized distributions for the signal templates corresponding to the three polarization contributions. In Figure 2 (right) the best fit of the signal and background template contamination is superimposed on the observed data. The signal and background yields for the used channels can be found in Ref. [7]. The same template fit is also performed in the dilepton channel, where the event is reconstructed taking the most likely solution after solving a set of six independent kinematic equations.

For the second method, information about the polarization is obtained from complementary 
observables. The angular asymmetries, $A_{+}$and $A_{-}$are defined as:

$$
A_{ \pm}=\frac{N\left(\cos \Theta^{*}>z_{ \pm}\right)-N\left(\cos \Theta^{*}<z_{ \pm}\right)}{N\left(\cos \Theta^{*}>z_{ \pm}\right)+N\left(\cos \Theta^{*}<z_{ \pm}\right)}
$$

with $z_{ \pm}= \pm\left(1-2^{2 / 3}\right)$ for $A_{ \pm}$, allowing the dependence on $F_{L}$ and $F_{R}$ to cancel, respectively [13]. After background subtraction the events are counted above and below the thresholds $z_{ \pm}$in a binned unfolded distribution of $\cos \Theta^{*}$.

The combination of the individual measurements of the $\mathrm{W}$ boson helicity fractions and asymmetries in the single-lepton and dilepton channels is done using the best linear unbiased estimator method [19]. The four measurements of the helicity fractions and the combined values are shown in Figure 3 (left). The observed polarization values of $F_{0}=0.67 \pm 0.07, F_{L}=0.32 \pm 0.04$ and $F_{R}=0.01 \pm 0.05$ are in good agreement with NNLO QCD predictions and are more precise than previous results [20]. In this analysis the main systematic uncertainties arise from the signal and background modelling, the influence of the jet energy scale and additional contributions coming from method-specific systematics like the background subtraction for the asymmetry method.
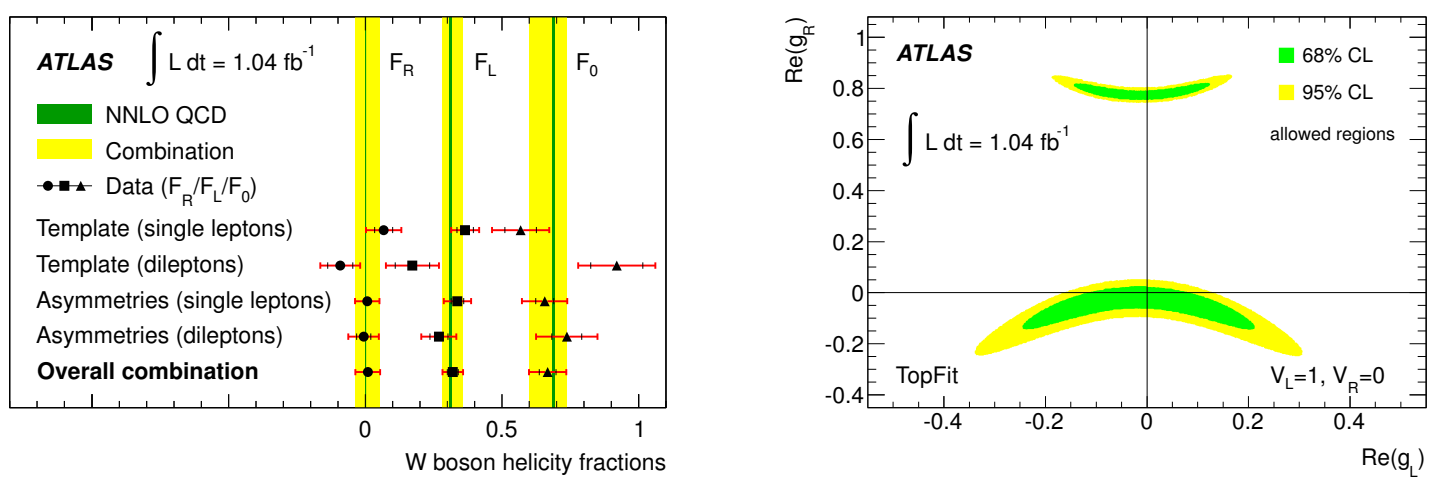

Figure 3: (Left) Overview of the four measurements of the $W$ boson helicity fractions and their combined values. (Right) Allowed regions at $68 \%$ and $95 \%$ conficence level (CL) for the $W t b$ anomalous couplings $g_{L}$ and $g_{R}[7]$.

Non-SM scenarios can yield anomalous $W t b$ couplings reflected in deviations of the helicity fractions and angular asymmetries from the SM calculations [16]. Such new interactions with the top quark may exist at higher energies. In effective field theories, dimension-six operators can be introduced which modify the $W t b$ vertex [17]. Limits on the $W t b$ vertex anomalous couplings are obtained from the combined results on the $W$ boson helicity fractions. Figure 3 (right) shows the allowed regions of the anomalous couplings $g_{L}$ and $g_{R}$ assuming $V_{R}=0$. Allowing only one nonvanishing contribution at a time one can also set limits on the individual terms. At $95 \%$ confidence level these are:

$$
\begin{aligned}
& \operatorname{Re}\left(V_{R}\right) \in[-0.20,0.23], \\
& \operatorname{Re}\left(g_{L}\right) \in[-0.14,0.11] \text { and } \\
& \operatorname{Re}\left(g_{R}\right) \in[-0.08,0.04] .
\end{aligned}
$$




\section{Conclusion}

These proceedings summarize the results of two top properties measurements, which are all consistent with the SM expectations. For the spin correlation measurement a hypothesis of zero spin correlation is excluded with a significance of 5.1 standard deviations. The results of the $\mathrm{W}$ helicity measurement are consistent with the (V-A) structure of the Wtb vertex and improve previously obtained limits on new physics contributions [21].

\section{References}

[1] F. Abe et al. [CDF Collaboration], Phys. Rev. Lett. 74, 2626 (1995).

[2] S. Abachi et al. [DØ Collaboration], Phys. Rev. Lett. 74, 2632 (1995).

[3] [Tevatron Electroweak Working Group], arXiv:1107.5255 [hep-ex].

[4] W. Bernreuther, J. Phys. G 35, 083001 (2008).

[5] W. Bernreuther and Z. -G. Si, Nucl. Phys. B 837, 90 (2010).

[6] ATLAS Collaboration, JHEP 1206, 088 (2012).

[7] ATLAS Collaboration, Phys. Rev. Lett. 108, 212001 (2012).

[8] T. Stelzer and S. Willenbrock, Phys. Lett. B 374, 169 (1996).

[9] T. Aaltonen et al. [CDF Collaboration], Phys. Rev. D 83, 031104 (2011); B. Abbott et al. [D0 Collaboration], Phys. Rev. Lett. 85, 256 (2000); V. M. Abazov et al. [DØ Collaboration], Phys. Lett. B 702, 16 (2011); V. M. Abazov et al. [DØ Collaboration], Phys. Rev. Lett. 107, 032001 (2011); V. M. Abazov et al. [DØ Collaboration], Phys. Rev. Lett. 108, 032004 (2012).

[10] G. Mahlon and S. J. Parke, Phys. Rev. D 81, 074024 (2010).

[11] P. Uwer, Phys. Lett. B 609, 271 (2005).

[12] P. M. Nadolsky et al., Phys. Rev. D 78, 013004 (2008).

[13] J. A. Aguilar-Saavedra et al., Eur. Phys. J. C 50, 519 (2007).

[14] A. Czarnecki, J. G. Korner, and J. H. Piclum, Phys. Rev. D 81, 111503 (2010).

[15] ATLAS Collaboration, JINST 3, S08003 (2008).

[16] G. L. Kane, G. A. Ladinsky, and C. P. Yuan, Phys. Rev. D 45, 124 (1992); J. A. Aguilar-Saavedra et al., Eur. Phys. J. C 50, 519 (2007).

[17] W. Buchmüller and D. Wyler, Nucl. Phys. B 268 (1986) 621; J. A. Aguilar-Saavedra, Nucl. Phys. B 812, 181 (2009); C. Zhang and S. Willenbrock, Phys. Rev. D 83, 034006 (2011).

[18] ATLAS Collaboration, arXiv:1203.4211;ATLAS Collaboration, arXiv:1203.5755.

[19] L. Lyons, D. Gibaut, and P. Clifford, Nucl. Instrum. Meth. A 270, 110 (1988); A. Valassi, Nucl. Instrum. Meth. A 500, 391 (2003).

[20] CDF and DØ Collaborations, arXiv:1202.5272, T. Aaltonen et al. [CDF Collaboration], Phys. Rev. Lett. 105, 042002 (2010); V. Abazov et al. [DØ Collaboration], Phys. Rev. D 83, 032009 (2011).

[21] V. Abazov et al. [DØ Collaboration], arXiv:1204.2332. 